\title{
ASPECTOS QUE INTERACTUAN EN EL PROCESO ADAPTATIVO ENTRE NIÑOS EN SITUACIÓN DE DESPLAZAMIENTO Y SUS NUEVOS ENTORNOS
}

\author{
INTERACTIVE ASPECTS IN THE ADAPTATION PROCESS \\ BETWEEN DISPLACED \\ CHILDREN AND THEIR NEW SURROUNDINGS
}

Venur Marina Morales López ${ }^{1}$ Yamile Guzmán Valencia Henry Granada Echeverry ${ }^{2}$

MORALES LÓPEZ, V.M.; GUZMÁN VALENCIA, Y.; GRANADA ECHEVERRY, H. Aspectos que interactúan em el proceso adaptativo entre niños em situación de desplazamiento y sus nuevos entornos. Rev. Bras. Cresc. Desenv. Hum., São Paulo, 2003.

Resumen: Este artículo describe la percepción organizada por un grupo de niños en situación de desplazamiento acerca de su relación con el ambiente actual y de los aspectos que la favorecen o la desfavorecen. Es un estudio exploratorio que analiza la autopercepción de los niños participantes, su relación con los mesosistemas anteriores y actuales y el rol familiar y escolar en esta relación, con base en la observación, los mapas cognitivos, las historias de vida y las entrevistas con padres y profesores. No todos los niños consideran el desplazamiento y el proceso adaptativo negativamente. Concluimos que no es posible generalizar la forma usualmente negativa, como los niños asumen su desplazamiento y su relación con los nuevos entornos.

Palabras-claves: niños desplazados; proceso adaptativo; ambiente ecológico; transición ambiental

\section{INTRODUCCIÓN}

En este artículo abordaremos un tipo específico de interacción psicoambiental en la que participan niños en situación de desplazamiento forzoso y sus entornos receptores, aún en proceso de construcción. Cabe anotar que el Fondo de las Naciones Unidas para la Infancia (UNICEF) en el año 2002, definió el desplazamiento como un tipo de migración involuntaria al interior de un mismo país en donde ciudadanos amenazados abandonan ya sea de forma individual, familiar o colectiva, sus sitios habituales de vivienda y trabajo como última estrategia para salvar sus vidas. En este caso nos referimos a un grupo especial de niños cuyas familias se vieron obligadas a huir de sus lugares de origen por diferentes amenazas y actualmente viven en un sector subnormal de la ciudad de Cali (suroccidente colombiano).

Ellos participaron en un estudio exploratorio que realizamos en el año 2002 de cuyos resultados surge este documento. Nuestra reflexión actual hace énfasis, por un lado, en las estrategias que los pequeños han organizado para interactuar con su nuevo ambiente las cuales pueden ser adaptativas o desadaptativas, dependiendo de los efectos que implica para

\footnotetext{
Departamento de Psicologia Social, Universidad del Valle, Cali, Colombia.

1 venurcita22@hotmail.com

2 granadahe@yahoo.com Henry Granada. Apartado Aereo 25534. Cali, Colombia.
} 
los niños y para los entornos en los que viven. Por otro lado, contempla la forma en que los ambientes favorecen o desfavorecen dichas estrategias.

A continuación, presentamos los aspectos generales del estudio realizado con los niños en cuanto a contexto, conceptos teóricos, estrategias metodológicas y resultados obtenidos.

\section{JUSTIFICACIÓN Y OBJETIVOS}

Cumpliendo con un requisito académico, en el año 2002 realizamos una investigación cualitativa sobre el proceso adaptativo entre niños en situación de desplazamiento y sus nuevos entornos, motivada por la falta de información rigurosa acerca de la problemática psicológica que genera el desplazamiento tanto en quienes lo sufren en carne propia como en la comunidad que se desarticula con su partida y en las comunidades que se desequilibran con su llegada. Especialmente en el caso de los niños quienes según la UNICEF (2001), constituyen más de la mitad de la población desplazada del país, encontramos que la mayoría de estudios existentes se limitan a abordar los efectos psicopatológicos que provoca el desplazamiento en la vida de los niños y no indaga sobre la forma en que los menores afrontan su realidad ni sobre las estrategias que organizan para seguir viviendo en los nuevos ambientes, por ello, los objetivos que guiaron nuestro trabajo fueron:

1) Conocer cómo perciben los niños en situación de desplazamiento, su interacción con el nuevo ambiente.

2) Explorar desde sus puntos de vista, cuáles son las fortalezas tanto en ellos como en los nuevos entornos que facilitan un proceso adaptativo exitoso.

\section{MARCO CONTEXTUAL}

Como ya dijimos, el desplazamiento forzoso de población es una problemática cada día más frecuente y generalizada en Colombia; de acuerdo con el último boletín de CODHES (consultoría para los derechos humanos y el desplazamiento, correspondiente al tercer trimestre de 2002), 149.387 personas fueron desplazadas; un promedio de 1.623 personas cada día, 67 personas cada hora y una familia cada 10 minutos. Bogotá D.C. fue la principal ciudad receptora con 17.500 personas.

A lo anterior, GONZÁLEZ (apud REYES, 2002) agrega que el desplazamiento es la peor catástrofe en la vida de las personas porque vulnera todos sus derechos y socialmente es una dinámica caótica de redistribución de la población en el territorio, y a nivel político, el desplazamiento es un sistema de exclusión que busca la homogeneización de la cultura. Otros autores, como RIVAS y GUTIÉRREZ (1996) Y ROJAS y ROMERO (2000) afirman radicalmente que el desplazamiento arranca los significados de la vida de las personas que lo sufren y de las comunidades que se desintegran con la partida.

En cuanto al sitio donde habitan los niños desplazados y sus familias, está ubicado en una zona de ladera de la comuna 18 de la ciudad de Cali (suroccidente de la ciudad), en un sector de antigua explotación minera que por su deterioro geológico ha sido considerada zona de alto riesgo; sus habitantes cuentan con poca cobertura de servicios básicos como agua potable, energía eléctrica, educación, salud, recolección de basuras, vías de transporte y de comunicación a lo que se suma la inseguridad social, el desempleo, la presencia de guerrilla y grupos de limpieza social además de los problemas ambientales como la contaminación por desechos, del aire y de las fuentes hídricas, así como los constantes deslizamientos que se suscitan con las lluvias por lo erosionado del terreno.

De acuerdo con las familias entrevistadas, sus mayores problemáticas actuales son la falta de vivienda propia, la descomposición social que afecta sobre todo a los niños y la falta de dinero para sufragar los gastos de la familia, como son la alimentación, el arriendo, la salud y la educación de los hijos. 


\section{CONCEPTOS CLAVES DEL MARCO TEÓRICO}

Teóricamente nos apoyamos en tres autores: BRONFENBRENNER (1987), KAMII (1987) y HOLAHAN (2001). HOLAHAN y BRONFENBRENNER conciben el proceso adaptativo como proceso dinámico y abierto, en donde tanto el hombre como el ambiente tienen una participación activa a través de la cual se construyen o se destruyen mutuamente.

De acuerdo con HOLAHAN (2001) en el proceso adaptativo intervienen tres unidades principales: el hombre, el ambiente y los procesos cognitivos mediadores. El autor define al sujeto como una persona íntegra, con una organización psicológica propia construído en interacción con el ambiente, a través de la cual percibe sus entornos los evalúa y diseña las estrategias de conducta para afrontar las condiciones de los mismos.

El ambiente ha sido denominado por BRONFENBRENNER (1987) como ambiente ecológico puesto que es un sistema en donde interactúan aspectos topográficos, sociales, culturales e históricos y plantea que dicho ambiente ecológico está compuesto por cuatro niveles o anillos concéntricos que son: microsistemas, formados por los entornos en que participa el individuo y poseen patrones específicos de roles y actividades. El segundo nivel es el mesosistema compuesto por las interac-ciones que se tejen entre estos microsistemas que entre más estrechas sean, más favorecen el proceso adaptativo. En tercer lugar, Bronfenbrenner menciona el exosistema que está formado por los entornos en los que no participa directamente el sujeto pero que afectan o son afectados por los microsistemas. Por último, este autor menciona el macrosistema el cual contiene los patrones culturales, es decir, los valores éticos y religiosos y los sistemas de creencias que dan forma a los tres niveles anteriores.

En cuanto a los procesos mediadores, según HOLAHAN (2001), son el conjunto de procesos cognitivos entre ellos la percepción ambiental, la cognición ambiental y las actitu- des ambientales que le permiten al sujeto percibir y evaluar los entornos de los que forma parte para tomar decisiones sobre la manera como va a comportarse en ellos. De esta forma el proceso adaptativo es una dialéctica entre el hombre y el ambiente mediada por los procesos cognitivos y las estrategias de conducta.

Otro aspecto vital en la interacción persona ambiente determinante para el desarrollo de cada una de las partes, se presentan cuando las personas viven un cambio ya sea de entornos, de roles, de actividades o de los tres al tiempo. Este hecho ha sido denominado por BRONFENBRENNER (1987) como transición ecológica, según el autor, la forma como la persona interprete y asuma estos cambios, influye enormemente en su proceso adaptativo. Este concepto aporta elementos para la comprensión de la realidad de los niños participantes puesto que ellos han sido objeto de una transición ecológica repentina e involuntaria en muchos casos.

Respecto a KAMII (1987), acuñamos principalmente su concepto de autonomía porque es un aspecto decisivo de la organización psicológica para que el sujeto logre construir una relación adecuada con sus entornos. De acuerdo con la autora, la autonomía tiene dos aspectos integrados entre sí: el intelectual y el moral. A nivel intelectual, la autonomía implica que el sujeto sea consciente del conocimiento que posee y pueda utilizarlo por sí mismo en el diseño de estrategias para la resolución de problemas; a nivel moral, el sujeto autónomo tiene un sentido ético de las normas y de sus responsabilidades consigo mismo y con los demás.

También tuvimos en cuenta los estudios realizados por AMAR (1999) y por PALACIO (1999) quienes concluyeron que los niños pueden sobreponerse a situaciones tan difíciles como la pobreza y el desplazamiento, logran ser felices, tener sueños y esperanzas a pesar de las circunstancias penosas de la vida. Los dos autores afirman que los niños varones son más resistentes a las tragedias que las niñas y PALACIO (op. cit.) plantea que hay factores en el ambiente que favorecen esa capacidad de 
resistencia que ha llamado resiliencia. Los aspectos protectores de la resiliencia psicológica en los niños víctimas de la violencia son: una actitud parental competente, buena relación por lo menos con uno de los padres, apoyo del entorno, buenas redes sociales y la educación.

Incluimos, además, un estudio realizado por MARTÍNEZ, MAYA y GARCÍA (2000), en donde analizaron el papel de las redes sociales en el proceso de integración sociocultural de los inmigrantes a la sociedad de llegada. Según estos autores, las redes sociales son vitales en la integración cultural porque proveen al inmigrante de apoyo emocional e instrumental para afrontar las dificultades de los nuevos entornos y al mismo tiempo les permite asimilar la nueva cultura de una manera práctica, paulatina y menos agresiva.

\section{METODO}

\section{Sujetos}

Además de los niños, en esta investigación participaron representantes de sus mesosistemas anterior y posterior al desplazamiento, como son los casos de las familias que han compartido con los niños ambos mesosistemas y los profesores de la escuela actual.

Los niños participantes son de origen campesino; en el momento de trabajar con ellos sus edades oscilaban entre los 8 y 12 años y fueron desplazados junto a sus familias por amenazas directas e indirectas de los grupos armados al margen de la ley que protagonizan el conflicto armado del país.

Estos menores y sus familias, residen en la comuna 18 de la ciudad de Cali, desde hace un año en zona de ladera. Presentan bajos niveles de escolaridad; sólo uno de ellos estaba terminando la primaria y algunos nunca habían asistido a la escuela. Antes de ser desplazados, se dedicaban a las labores del campo como cultivar y cuidar los animales, actividad que los satisfacía porque tenían un contacto directo con la naturaleza. Así mismo jugaban, estudiaban (cuando tenían maestros) y mientras que algunos se podían dedicar a estas dos últimas labores, otros tenían que trabajar tiempo completo como los adultos.

Actualmente, la mayoría de estos niños se dedican a estudiar en las mañanas, a trabajar en las tardes como vendedores ambulantes o realizando las labores domésticas mientras sus padres trabajan. En total trabajamos con 25 niños pero elegimos sólo a 10 , conjuntamente con sus respectivas familias y escuelas para realizar el informe final.

El siguiente cuadro (Cuadro 1) ilustra las principales características de los cinco participantes cuyos registros aparecerán a lo largo del artículo.

En cuanto a las familias, en total entrevistamos a seis puesto que contamos con dos grupos de tres hermanos cada uno en el conjunto de los 10 niños participantes; éstas familias al igual que sus hijos son campesinas, antes de su desplazamiento convivían con la familia extensa pero en el nuevo entorno sólo lo hacen con los miembros de la familia nuclear. En general, son grupos familiares numerosos, están compuestos por los padres y entre 4 y 8 hijos; los niveles de escolaridad no rebasan la primaria, son familias jóvenes, ninguno de los adultos llega a los 40 años.

Antes de ser desplazadas, se dedicaban a cultivar sus tierras y a atender sus negocios, eran autosuficientes, ahora sólo trabajan uno o dos miembros de la familia contando con los niños, realizando actividades como el reciclaje, el oficio doméstico, la construcción y las ventas ambulantes que no les ofrecen ninguna estabilidad laboral y económica, por lo tanto, deben subsistir con el poco dinero que obtienen. Sin embargo, también existen grupos familiares que han logrado obtener créditos por medio de instituciones humanitarias, organizar su negocio en la casa donde pagan arriendo y tener una vida más cómoda. Así mismo, entrevistamos a los profesores de las dos escuelas a las que asisten los niños participantes que están estudiando Estas escuelas son las más con- 
CUADRO 1. PRINCIPALES CARACTERÍSTICAS DE LOS NIÑOS Y DE SUS ENTORNOS

\begin{tabular}{|c|c|c|c|c|c|c|c|c|c|c|c|}
\hline \multirow[b]{3}{*}{ 㺃 } & \multirow{2}{*}{\multicolumn{5}{|c|}{ características sociodemográficas }} & \multicolumn{6}{|c|}{ aspectos generales de los ambientes } \\
\hline & & & & & & \multicolumn{3}{|c|}{ Ambiente Anterior } & \multicolumn{3}{|c|}{ Ambiente Actual } \\
\hline & 롱 & 苟 & 产 & 흘률 & 这 & 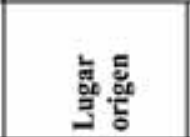 & 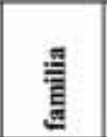 & 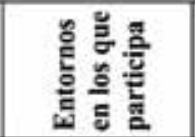 & 䑁 & 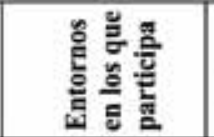 & 造造 \\
\hline G & 12 & $\mathrm{M}$ & $1^{\circ}$ primaria & $\begin{array}{c}\text { Afrocolom- } \\
\text { Biano }\end{array}$ & $\begin{array}{l}\text { Amenazas } \\
\text { indirectas } \\
\text { a la } \\
\text { familia }\end{array}$ & $\begin{array}{l}\text { López de Micay } \\
\text { zona runal del } \\
\text { Cauca }\end{array}$ & Nuclear & $\begin{array}{l}\text { Familiar, escolar } \\
\text { y vecinal }\end{array}$ & Nuclear & $\begin{array}{l}\text { Familiar, escolar, } \\
\text { amigos laboral y } \\
\text { vecinal }\end{array}$ & $\begin{array}{l}\text { Quiere ser } \\
\text { doctor }\end{array}$ \\
\hline $\mathrm{N}$ & 8 & M & Ninguno & $\begin{array}{c}\text { Afrocolom- } \\
\text { Biano }\end{array}$ & \begin{tabular}{l|} 
Amenazas \\
indirectas \\
contra la \\
familia \\
\end{tabular} & $\begin{array}{l}\text { Honduras zona } \\
\text { rural del Cauca } \\
\text { Suroccidente } \\
\text { colombiano }\end{array}$ & Extensa & $\begin{array}{l}\text { Familiar y } \\
\text { entorno vecinal }\end{array}$ & Nuclear & $\begin{array}{l}\text { Familiar, amigos } \\
\text { y entorno laboral }\end{array}$ & $\begin{array}{l}\text { Trabajar } \\
\text { en una } \\
\text { oficina }\end{array}$ \\
\hline C.M & 9 & $\mathbf{F}$ & $1^{\circ}$ primaria & Mestizo & $\begin{array}{l}\text { Amenazas } \\
\text { indirectas } \\
\text { a la } \\
\text { familia }\end{array}$ & $\begin{array}{l}\text { Zona rural } \\
\text { de San Pedro } \\
\text { Valle }\end{array}$ & Extensa & $\begin{array}{l}\text { Familiar, } \\
\text { amigos y } \\
\text { vecinal }\end{array}$ & Nuclear & $\begin{array}{l}\text { Familiar, escolar } \\
\text { y vecinal }\end{array}$ & $\begin{array}{l}\text { Desea ser } \\
\text { modelo }\end{array}$ \\
\hline E.J & 11 & $\mathbf{F}$ & $3^{\circ}$ primaria & Mestizo & $\begin{array}{l}\text { Separación } \\
\text { de los } \\
\text { Padres }\end{array}$ & $\begin{array}{l}\text { Caloto zona } \\
\text { rural del cauca }\end{array}$ & Extensa & \begin{tabular}{|l|} 
Familiar, \\
escolar, amigos, \\
vecinal y \\
religioso
\end{tabular} & Nuclear & $\begin{array}{l}\text { Familiar, escolar, } \\
\text { amigos vecinal y } \\
\text { religioso }\end{array}$ & $\begin{array}{l}\text { Quiere ser } \\
\text { doctora }\end{array}$ \\
\hline w & 11 & M & $5^{\circ}$ primaria & Mestizo & \begin{tabular}{l|} 
Amenazas \\
Indirectas \\
a la \\
familia
\end{tabular} & $\begin{array}{l}\text { San Carlos } \\
\text { zona rural de } \\
\text { Antioquia, } \\
\text { noroccidente } \\
\text { colombiano }\end{array}$ & Nuclear & $\begin{array}{l}\text { Familiar, } \\
\text { escolar, amigos, } \\
\text { vecinal, } \\
\text { religioso y } \\
\text { laboral del padre }\end{array}$ & Nuclear & $\begin{array}{l}\text { Familiar, escolar, } \\
\text { vecinal y } \\
\text { religioso }\end{array}$ & $\begin{array}{l}\text { Sueña con } \\
\text { ser piloto }\end{array}$ \\
\hline
\end{tabular}

curridas por todos los niños desplazados y no desplazados de la zona ya que son muy escasos los centros educativos en el sector, una de ellas pertenece al Estado y la otra a una organización comunitaria.

La estatal está mejor dotada, cuenta con más espacios de juego para los niños, más materiales didácticos y un buen número de profesores; la segunda es más pequeña, con menos dotación, menos personal, pero está más integrada a la cotidianidad de los niños, sus familias y sus comunidades. Las dos escuelas prestan servicio de restaurante para los niños y les ofrecen alternativas de entretenimiento extracurriculares como danzas, teatro, clases de percusión, etc.

\section{Procedimiento}

Con respecto a las estrategias metodológicas, el estudio se llevó a cabo en tres etapas: en la primera, se realizó la revisión bibliográfica, contactamos la comunidad que participó, efectuamos las primeras observaciones naturales a los niños y diseñamos los instrumentos de recolección de datos : las historias de vida, los mapas cognitivos y la observación del comportamiento. Para el trabajo con las familias y los profesores realizamos dos tipos de entrevistas. Los instrumentos diseñados para el trabajo con los niños son complementarios entre sí y fueron diseñados tanto, para recoger información como para ayudarles a construir una visión positiva de sí mismos y de sus entornos, para ello se utilizaron varias estrategias como el dibujo, el juego y la elaboración de maquetas, que por un lado, motivaron a los niños y que por otro, desencadenaron una dinámica grupal de intercambio entre ellos, muy favorable para el proceso. Particularmente, los mapas cognitivos constituyeron la técnica central del estudio porque nos permitieron conocer las interpretaciones y las representaciones mentales que los niños han construido de sus ambientes anteriores y posteriores al desplazamiento, y de su relación con ellos. Los niños plasmaron sus mapas cognitivos de cada uno de sus ambientes en maquetas que elaboraron con diferentes materiales reciclables y vegetales. Para 
codificar la información contenida en dichas maquetas, diseñamos el siguiente sistema de categorías:

Tipo de mapa cognitivo graficado, ésta categoría está compuesta por las siguientes cuatro clasificaciones:

a) Gráfico armónico: Se refiere a la maqueta que refleja los siguientes tres aspectos:

1 - Elementos que simbolicen comunicación y articulación entre los diferentes componentes gráficos de la maqueta; estos elementos pueden ser: una equilibrada distribución del espacio; presencia de vías de transporte o de comunicación.

2 - Elementos que muestren posibilidades que los niños han encontrado para sus vidas en los entornos graficados además de la vivienda, estos pueden ser, canchas de fútbol, piscinas, escuelas u otros espacios de recreación, los cuales favorecen actividades como el estudio, el juego, la interacción social y el disfrute del entorno, que son relevantes para el desarrollo psicológico normal de todo niño.

3 - Presencia expresa de figuras humanas en la maqueta como signo de identificación del niño o que se pueda constatar a través de la observación que el niño realizó la maqueta como una narración de su vida cotidiana.

b) Gráfico con cierto grado de relación positiva o negativa: esta subcategoría incluye aquellos gráficos de mapas cognitivos que solo muestran dos de los tres elementos que componen la representación armónica descritos anteriormente y se considera negativo el grado de relación cuando los gráficos además muestran ambientes limitados o contienen signos agresivos como armas, muertos, etc.
3 - Os desenhos apresentados a seguir foram originalmente realizados com lápis coloridos.

c) Gráfico desarticulado y ajeno: está subcategoría incluye aquellas maquetas que no reflejan ninguno de los tres elementos antes citados (articulación de los elementos que componen la maqueta, presencia de figura humana y localización de recursos para la vida).

d) Gráfico fantaseado: se refiere a modelos de mapas cognitivos que muestran un alto grado de distorsión con el entorno real provocada por los rasgos de personalidad del sujeto.

En la segunda etapa, llevamos a cabo el trabajo directo con los niños a través de una serie de talleres en los que ellos participaron desde sus necesidades, inquietudes y expectativas. También se llevaron a cabo las visitas a cada una de las familias en su domicilio para aplicar las entrevistas; lo mismo sucedió con las dos escuelas.

Durante la tercera etapa, afinamos los sistemas de categorías e iniciamos el proceso de análisis e interpretación de los datos obtenidos.

\section{RESULTADOS YANÁLISIS}

En éste apartado reseñamos los elementos que se ponen en juego en el proceso adaptativo, haciendo un análisis comparativo entre la interacción construida por los niños con su ambiente anterior y la que construyen con su ambiente actual. Ésta comparación muestra el papel que el niño ha jugado en su interacción con el ambiente y los efectos del desplazamiento sobre el proceso adaptativo.

A siguir, aparecen graficadas las maquetas $^{3}$, elaboradas por los cinco niños elegidos para el presente documento y a continuación la tabla que da cuenta de los aspectos que favorecen o dificultan la relación de los niños con sus nuevos entornos (Cuadro 2).

3 Os desenhos apresentados a seguir foram originalmente realizados com lápis coloridos. 


\section{CUADRO 2.ASPECTOS PSICOLÓGICOS,ACTITUDINALES Y CONDUCTUALES QUE FACILITAN O DIFICULTAN LAADAPTACIÓN DE LOS NIÑOS ALOS NUEVOS ENTORNOS}

\begin{tabular}{|c|c|c|}
\hline \multicolumn{2}{|c|}{$\stackrel{\mathscr{a}}{\rightleftarrows}$} & 1. C.M. \\
\hline \multirow{9}{*}{ 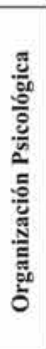 } & \multirow{5}{*}{ 魷 } & $\begin{array}{l}\text { Nivel adecuado de autonomía, tolerancia a la frustración, buena disposición para las relaciones sociales y construcción } \\
\text { de un proyecto de vida viable en el ambiente actual. }\end{array}$ \\
\hline & & $\begin{array}{l}\text { Nivel adecuado de autonomía, construcción de un sueño para el futuro acorde a las condiciones al nuevo ambiente y } \\
\text { disposición para las interacciones sociales. }\end{array}$ \\
\hline & & Sentimiento de autocuidado y de mejorar sus condiciones de vida. \\
\hline & & Buen nivel de autonomia y relaciones sociales limitadas a su grupo racial \\
\hline & & Nivel adecuado de autonomía y proyecto de vida acorde acorde con el ambiente \\
\hline & \multirow{4}{*}{ 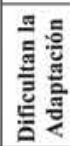 } & Estado psicológico depresivo, estado de ánimo predominantemente melancólico y desinterés por sus propias cosas. \\
\hline & & Nivel bajo de autonomía y estado psicológico depresivo \\
\hline & & $\begin{array}{l}\text { Estado psicológico depresivo, la no organización de su proyecto de vida de acuerdo a las condiciones de su ambiente } \\
\text { actual. }\end{array}$ \\
\hline & & Inestabilidad emocional \\
\hline \multirow{10}{*}{ 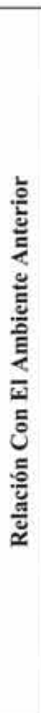 } & \multirow{5}{*}{ 产 } & $\begin{array}{l}\text { Valoración positiva de todos los elementos que constituyen este ambiente, la redes sociales, el ambiente físico-natural, } \\
\text { las diferentes actividades que tanto ella como los que la rodeaban desarrollaban en él. Realización de actividades que le } \\
\text { resultaban placenteras como el juego, visitar a la abuela y jugar con los primos. Asumir las responsabilidades } \\
\text { correspondientes que consistian en ayudar al aseo de la vivienda. }\end{array}$ \\
\hline & & $\begin{array}{l}\text { Establecimiento de relaciones estrechas com los miembros de la familia nuclear y extensa (a excepción del padre), con el } \\
\text { sistema de pares, del entorno vecinal, con los profesores y los compañeros de clase. El asumir responsabilidades tanto en } \\
\text { la familia como en la escuela positivamente. Preferencia por los espacios de juego y recreación a campo abierto. El } \\
\text { disfrute de las actividades que realiza con todo el grupo familiar como festejar cumpleaños, salir al pueblo e ir a misa los } \\
\text { domingos. }\end{array}$ \\
\hline & & Ninguno \\
\hline & & $\begin{array}{l}\text { Valoración positiva de todos los elementos que constituyen este ambiente: alternar su trabajo de campo con actividades } \\
\text { de recreación. Establecimiento de relaciones estrechas con los pares y con los adultos que comparten sus entornos. } \\
\text { Participación activa y gratificante en varios entornos que favorecen su desarrollo, como el familiar, el escolar y el } \\
\text { comunitario. }\end{array}$ \\
\hline & & $\begin{array}{l}\text { Evaluación positiva de todas las actividades que realiza en este entomo relacionadas con el juego y con el estudio. } \\
\text { Disfrute de las actividades que realizaba con la familia . valoración positiva de la mayoria de elementos que componen } \\
\text { este ambiente. Participación activa y gratificante en varios entornos que favorecen su desarrollo como la escuela, la } \\
\text { familia o el entorno vecinal. }\end{array}$ \\
\hline & \multirow{5}{*}{ 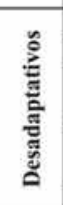 } & \\
\hline & & Percepción negativa de la interacción con el padre por maltrato físico y psicológico. \\
\hline & & $\begin{array}{l}\text { Tiene percepción negativa de la mayor parte de los elementos que componían el ambiente rural. No percibe ninguna } \\
\text { posibilidad para él en este ambiente. }\end{array}$ \\
\hline & & $\begin{array}{l}\text { La percepción de la escuela em este ambiente como un entorno ineficaz y no vislumbrar posibilidades de tener un buen } \\
\text { futuro por el aislamiento geográfico de sus entornos. }\end{array}$ \\
\hline & & Percepción de la escuela como el factor que obstaculiza la realización del proyecto de vida en este ambiente. \\
\hline \multirow{10}{*}{ 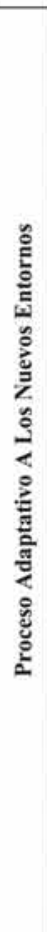 } & \multirow{5}{*}{ 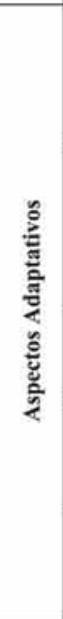 } & $\begin{array}{l}\text { Participación en varios entornos importantes para el desarrollo como son la familia, escuela, entorno vecinal. } \\
\text { Establecimiento de relaciones estrechas en los entornos que comparte tanto con pares como con adultos. Valoración de } \\
\text { la escuela como elemento positivo del ambiente actual porque alli le enseñan muchas cosas, porque los compañeros la } \\
\text { quieren y por los espacios fisicos para el juego. El asumir positivamente sus responsabilidades en la escuela y en la casa. } \\
\text { Alternar sus actividades obligatorias con actividades placenteras como escuchar música, bailar y ver tv. }\end{array}$ \\
\hline & & $\begin{array}{l}\text { Percepción adecuada del ambiente actual de la que concluye que en el ambiente anterior tenia más comodidades } \\
\text { materiales, pero era maltratada. En le ambiente actual, tiene pocas cosas pero nadie la trata mal. Participación activa en } \\
\text { actividades favorables para el desarrollo como la escuela, el sistema de pares, la lglesia y el trabajo de la madre. El } \\
\text { equilibrar actividades que hace por obligación con actividades que hace por placer como jugar, ver tv y escuchar música. } \\
\text { Establece vinculos con la redes sociales de dichos entornos. El disfrute de las actividades que realiza con la familia } \\
\text { como el aseo de la casa, ir a misa ver tv y platicar. }\end{array}$ \\
\hline & & $\begin{array}{l}\text { Percepción positiva de la mayor parte de los elementos que conforman el ambiente actual, sobre todo por las } \\
\text { posibilidades que le ofrece la cultura urbana, por la oportunidad de entrar a la escuela y de integrarse al nuevo sistemade } \\
\text { pares. Percepción positiva del desplazamiento. }\end{array}$ \\
\hline & & $\begin{array}{l}\text { Valoración muy positiva de la escuela por la enseñanza, por la posibilidad de establecer relaciones estrechas con sus } \\
\text { profesores y otros niños y por los espacios de juego que ofrece. Fortalecimiento delas relaciones intrafamiliares, disfrute } \\
\text { de las actividades realizadas con todo el grupo familiar. Aprovechar las posibilidades que ofrece la cultura urbana para la } \\
\text { recreación y para desarrollar las actividades laborales. }\end{array}$ \\
\hline & & $\begin{array}{l}\text { Valoración positiva de la escue la por su calidad académica vital para el proyecto de vida (ser piloto de la fuerza aérea } \\
\text { colombiana). Integración en el sistema de pares, escolar y vecinal y establecimiento de interacciones estrechas con sus } \\
\text { profesores y con sus vecinos. Fortalecimiento de las relaciones familiares. Disfrute de todas las posibilidades } \\
\text { académicas que ofrece la cultura urbana y de las actividades que realiza con su grupo familiar. }\end{array}$ \\
\hline & \multirow{5}{*}{ 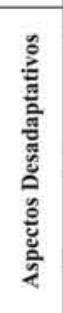 } & El conservar una relación muy estrecha con su ambiente anterior. \\
\hline & & $\begin{array}{l}\text { No ha podido elaborar la pérdida de su padre y aceptarlo como su agresor. Percepción negativa del mesosistema actual, } \\
\text { sobre todo el entorno laboral de la madre y las calles lejanas del barrio porque escucha balaceras cuando duerme. } \\
\text { Percepción negativa del diseño fisico urbano como una cárcel atestada de edificios y cemento, en comparación con los } \\
\text { bellos paisajes de su tierra. }\end{array}$ \\
\hline & & Construcción de relaciones lejanas con la familia nuclear \\
\hline & & $\begin{array}{l}\text { El asumir la responsabilidad de ser uno delos mayores soportes económicos de la familia desarrollando actividades } \\
\text { como: ventas ambulantes y malabarismo en los semáforos o pedir limosna. Percibe la carencia económica como un } \\
\text { limitante para vivir en este nuevo entorno. }\end{array}$ \\
\hline & & $\begin{array}{l}\text { Nostalgia por las posibilidades de interacción social de juego y por la comodidad y belleza del espacio fisico que } \\
\text { disfrutaba en el ambiente anterior. }\end{array}$ \\
\hline
\end{tabular}


PARTICIPANTE No. 6: N (8 años) Mapa cognitivo de su ambiente anterior.

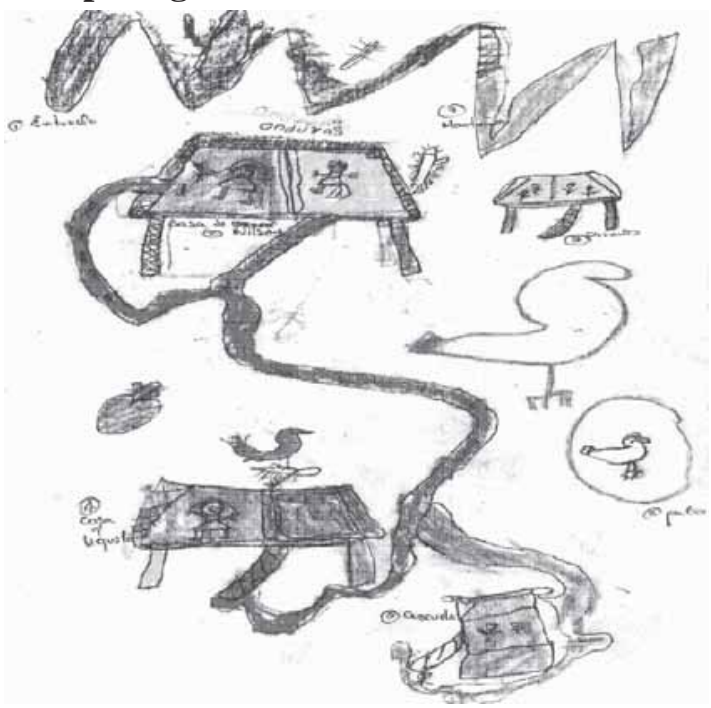

Ambiente anterior:

Hice la casa donde vivía con ellos, la familia de mi papá, también hice gente, patos, la escuela que también tiene gente afuera, la casa bonita, animales, una piña y niñitos por el camino hacia la escuela. De acá no se que me gusta, nada, y lo que menos me gusta es el trabajo porque es muy duro, me tocaba cargar agua y cargar leña y el revuelto ${ }^{4}$ desde muy lejos, eso era muy pesado y hacía mucho sol. No se que es lo que más extraño de allá.

\section{PARTICIPANTE No.8: G (12 años) Mapa cognitivo de su ambiente anterior}

Ambiente anterior:

Hice unas montañas que tienen árboles alrededor, esa es la entrada al pueblo de Nayita, acá hay una carretera que da la vuelta a todo el pueblo pero tiene desvíos para la casa donde yo vivo que es ésta (señala), también hice la casa de mis primos, otra casa que hay por allá cerca que es como la Iglesia y más abajo hice dos escuelas, una queda más lejos que la otra. Lo que más me gusta es la belleza del paisaje, que tenía mucho espacio para salir a caminar y que teníamos mucha comida. Lo que menos me gustaba es que no había transporte para sacar las cosechas, allá trabajaba en la finca, a veces jugaba con otros niños e iba a la escuela. Lo que más extraño es la comida, los ríos y los animales.
PARTICIPANTE No. 6: N (8 años) Mapa cognitivo de su ambiente actual.

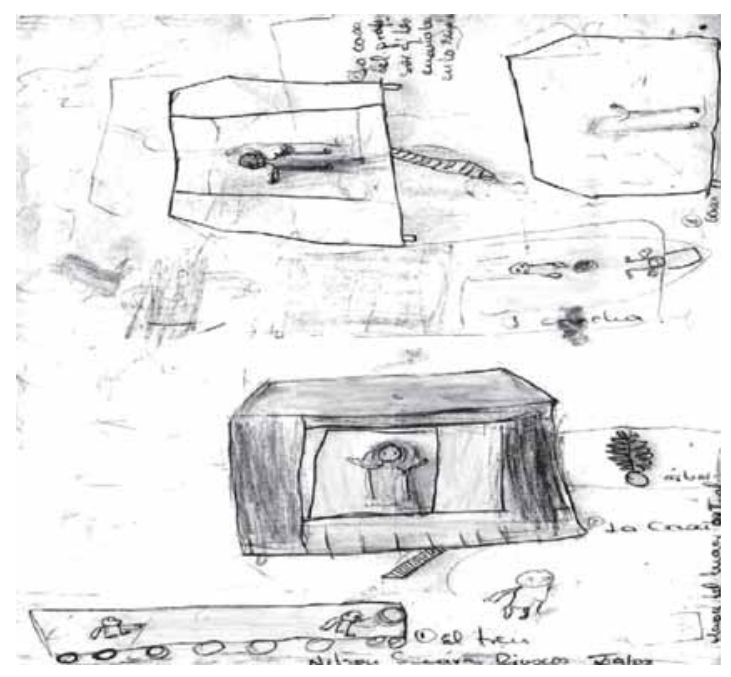

Ambiente actual:

Hice la casa, los árboles, otra casa, una cancha, un balón, una niña, los carros, el televisor en mi casa; lo que más me gusta de acá es las calles, los carros, la electricidad y lo que menos me gusta es la basura que hay botada en las calles. Yo prefiero vivir acá donde estoy ahora porque no me toca trabajar tanto.

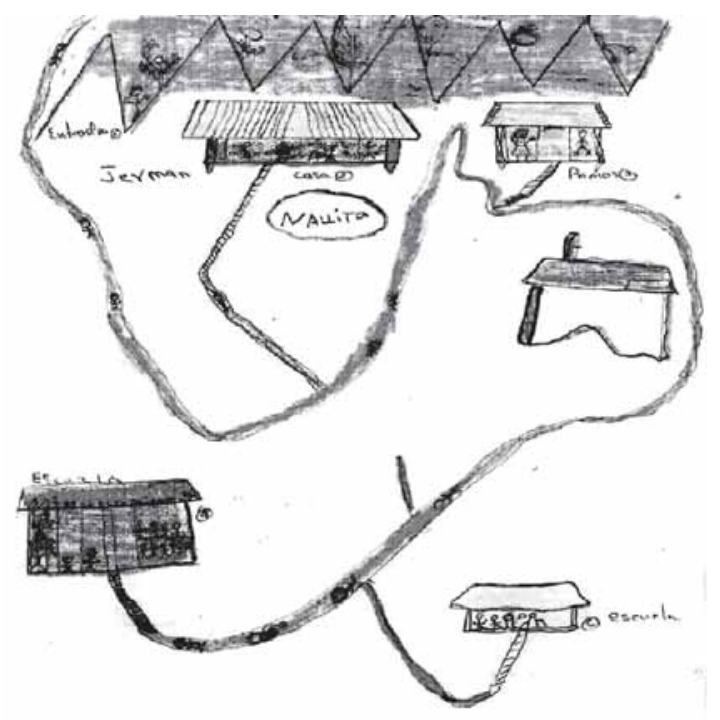

Ambiente actual:

Hice la casa de mis primos y el camino que baja a mi casa, las dos son de dos pisos; en

4 Plátanos, yuca y otros productos de pancoger. 
la casa de arriba, la de mis primos, tiene dos piezas, allí dibujé a mis primas; en la de abajo está mi mamá, también hay dos piezas, aquí arriba hay un árbol, debajo del árbol unas nubes y está lloviendo; al lado de la carretera hay otro árbol, luego una casita, por acá está la escuela (CECUCOL) que no me quedó bien hecho, y de último hay un carro de la policía y un niño al pie de un rifle. Lo que más me gusta es la energía $a^{5,}$ los carros, los edificios y los parques de atracciones mecánicas y lo que menos me gusta es que uno aquí pasa mucho trabajo para conseguir la comida y del arriendo, y la casa donde vivo tampoco me gusta porque es muy fea y está en muy mal sitio. Acá vengo a la escuela, voy al semáforo a trabajar y juego fútbol con los muchachos o con los primos. No sé cual de los dos sitios me gusta más porque acá uno vive muy bien por todas las cosas que hay, pero lo grave es que no se consigue ni para la comida ni para el arriendo. Cuando sea grande quiero ser soldado.

PARTICIPANTE No.8: G (12 años) Mapa cognitivo de su ambiente actual

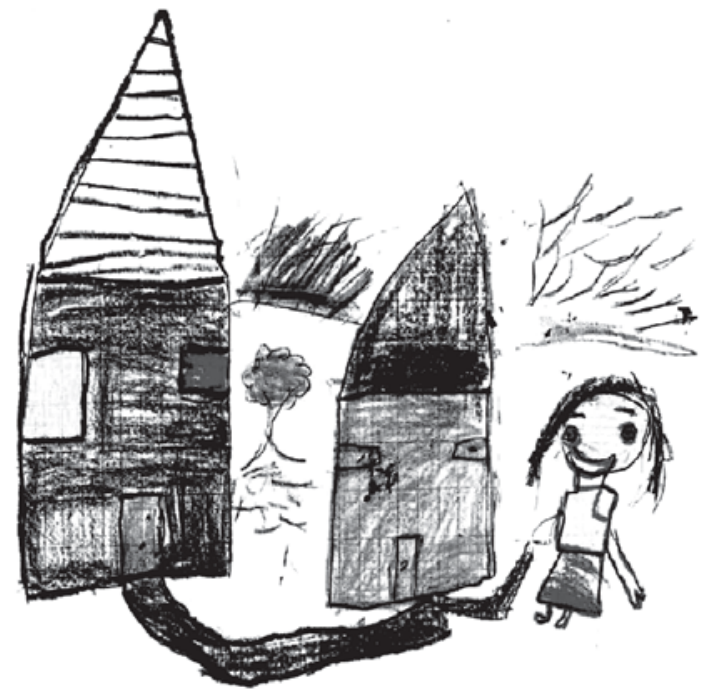

Ambiente anterior

Hice mi casa, la casa de mi abuelita, el camino que hay por ahí, las matas del jardín de mi tía, ya no más. Lo que más me gusta de acá, todo; allá yo jugaba y a veces le ayudaba a hacer oficio a mi mamá que yo podía hacer. Lo que más extraño de allá es mi abuelita.
PARTICIPANTE No. 2: CM (9 años) Mapa cognitivo de su ambiente anterior

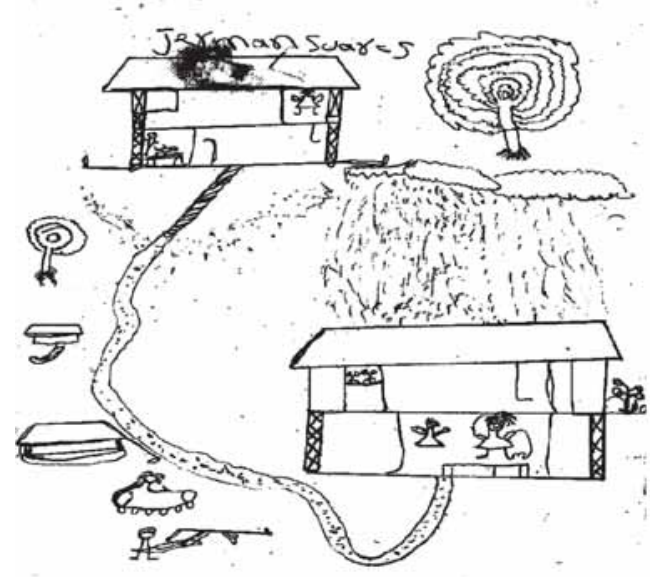

PARTICIPANTE No. 2: CM (9 años) Mapa cognitivo de su ambiente actual.
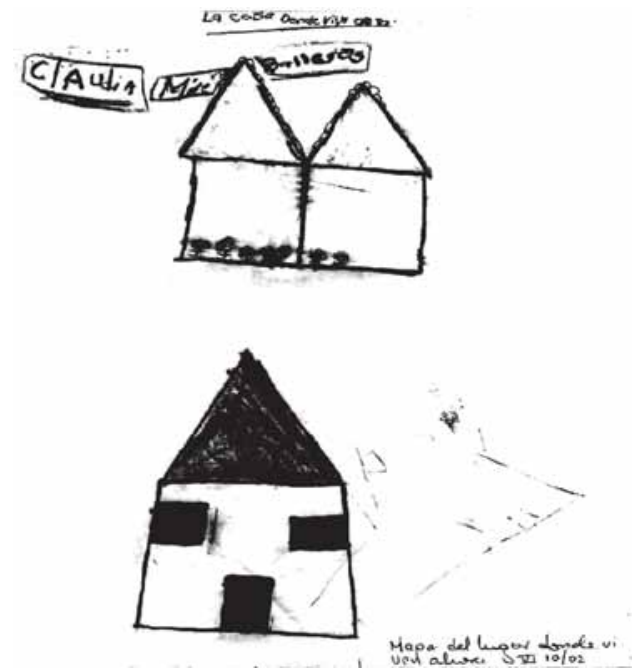

Ambiente actual:

Hice mi casa, la del frente de la vecina, el esposo de la vecina que está en la puerta, mi casa la hice de dos pisos y aquí dibujé a mis hermanitos (señala), la Escuela queda muy lejos por eso no la hice. Lo que más me gusta es la Escuela porque enseñan muchas cosas, porque hay muchos niños y porque es grande y bonita y lo que menos me gusta es que cuando llueve se hace mucho pantano. Aquí estudio, me toca hacer oficio, escucho música y a veces salgo al parque con mi mamá. Prefiero volver a la finca porque es muy bonita y hay mucho espacio para jugar. Cuando sea grande quiero ser bailarina.

5 Se refiere a la corriente eléctrica. 
PARTICIPANTE No. 3: EJ (11 años) Mapa cognitivo de su ambiente anterior

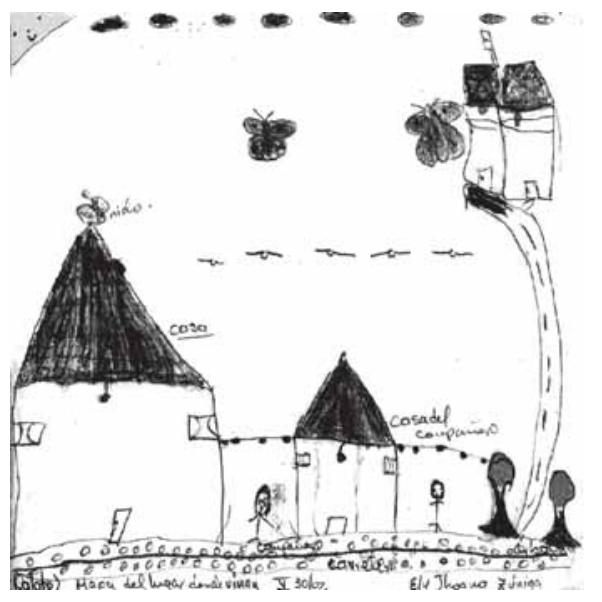

Ambiente anterior:

Hice mi casa que está más grande y arriba le hice un nido de pajaritos, hice la finca de mis vecinos y los animales de la casa, hice el camino, hice la escuela, hice los árboles, aquí me hice yo (señala), hice mi amiga y la casa de mi amiga, hice el sol, las nubes y los pajaritos. Lo que más me gustaba de allá era mi casa porque era grande y tenía muchos espacios para jugar, también me gustaba coger mangos con los muchachos en los árboles y también me gustaba ir a bañarme al río, también me gustaba mucho la pieza de mi hermana porque tenía muchos dibujos de paisajes muy bonitos. Lo que menos me gustaban eran las peleas de mis papás porque mi papá le pegaba a mi mamá y a nosotros nos mantenía echando. Lo que más extraño de allá es a mis papás y a mis hermanos. Allá sólo estudiaba y jugaba.

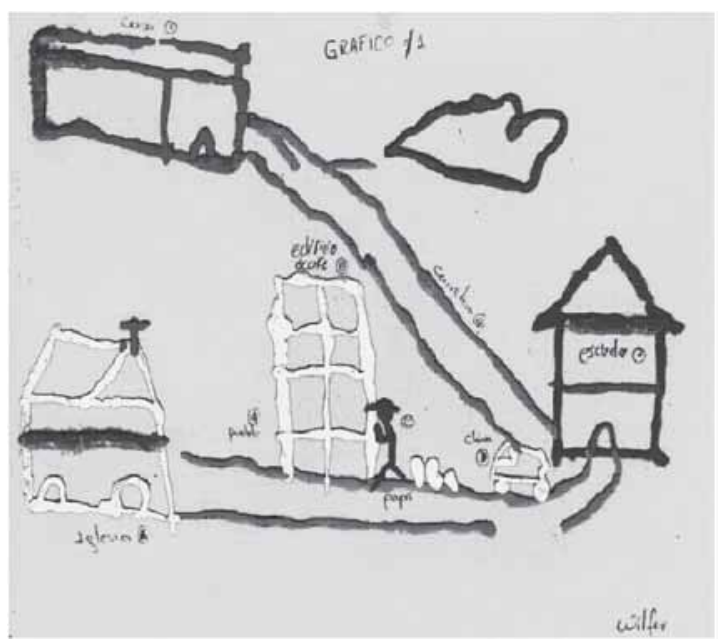

PARTICIPANTE No. 3: EJ (11 años) Mapa cognitivo de su ambiente actual

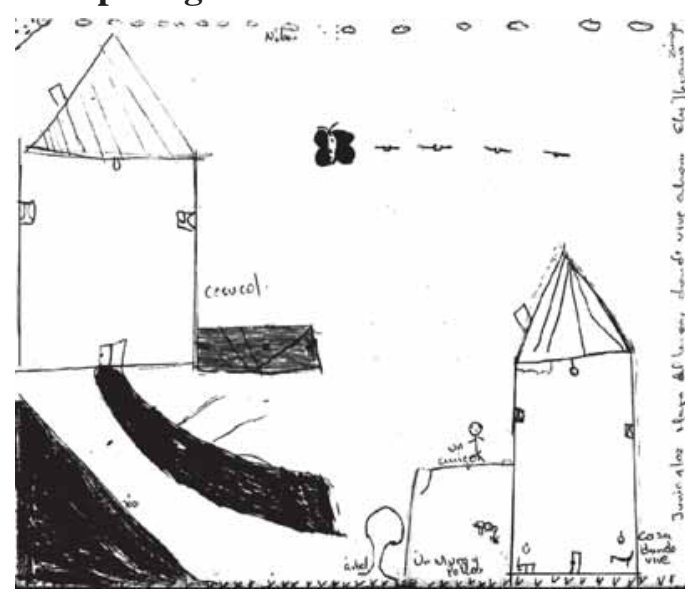

Ambiente actual:

Hice mi casa, los pajaritos, CECUCOL (la escuela), la casa de un amigo, un pollito de los que tenemos en la casa y un muro, y arriba está mi amigo; hice el río que pasa por aquí y el sol. Aquí lo que más me gusta es que aunque estoy viviendo en un ranchito vivo tranquila porque nadie nos está humillando ni echando, me gusta mucho el frente de mi casa porque ahí juego con mis vecinitos y me gusta mucho la escuela porque los profesores me tratan bien, puedo hacer muchas cosas como danza, teatro y el almuerzo sólo me vale cien pesos (\$100). Lo que menos me gusta es la inseguridad y los ladrones porque ya nos han robado varias veces, a mi mamá cuando venía del trabajo y un día también se metieron a la casa pero nosotros no estábamos en la casa. Aquí lo que más me gusta hacer es estudiar, jugar y me toca que ayudar a hacer el oficio de la casa y ayudarle a mi mamá en el trabajo cuando hace aseo. Yo prefiero quedarme aquí porque vivo tranquila. Cuando sea grande quiero ser veterinaria, a mi siempre me ha gustado mucho cuidar los animales y más por acá que hay muchos perritos tirados en la calle.

\section{PARTICIPANTE No. 9: W (11 años) Mapa cognitivo de su ambiente anterior}

Ambiente anterior

Acá hice la casa (señala), acá hice la escuela, acá hice la carretera, por acá hice la chiva que va para el pueblo y acá abajo hice el pueblo, hice la iglesia, el edificio del café, hice a mi papá. 
PARTICIPANTE No. 9: W (11 años) Mapa cognitivo de su ambiente actual

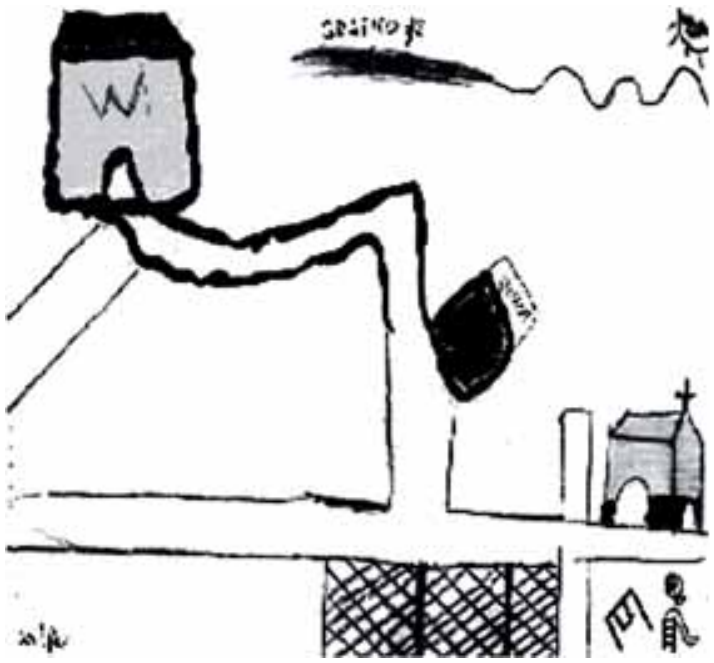

Ambiente actual

Este es cecucol (señala), acá está la iglesia, acá está la escuela, esta es la subida para el hospital, ésta es la casa que se alcanza a ver allá arriba. Aspectos psicológicos, actitudinales y conductuales que facilitan o dificultan la adaptación de los niños a los nuevos entornos.

El Cuadro 2 ilustra la interacción entre los diferentes elementos que conforman el proceso adaptativo a los nuevos entornos. Por un lado, encontramos los rasgos psicológicos principales de los niños y su interacción con el entorno anterior que constituyen el equipaje con el que el niño se relaciona con el nuevo ambiente; por otro lado, observamos el proceso adaptativo actual de los niños compuesto por sus estrategias de adaptación y por las posibilidades que le ofrecen los nuevos entornos.

Con respecto a la organización psicológica el cuadro muestra los aspectos que facilitan y los que dificultan la adaptación. Entre los facilitadores encontramos la autonomía, la disposición para las relaciones sociales, los proyectos de vida viables en el entorno actual y el sentimiento de protección de los niños ha- cia sí mismos. Estos facilitadores indican un buen nivel de desarrollo en los niños y por tanto una buena capacidad de interpretar en forma adecuada sus relaciones con el ambiente a través de los procesos mediadores y garantizan hasta cierto punto una adaptación exitosa porque son niños que tienen facilidad para resolver sus problemas en su relación con el entorno para asumir sus responsabilidades consigo mismo y con los demás para integrarse en las redes sociales que constituyen los nuevos entornos lo cual, de acuerdo con MARTÍNEZ, MAYA y GARCÍA (2000), es vital para la integración del nuevo orden social.

También, la mayoría de éstos niños tienen un sueño que orienta sus vidas, que los motiva a salir adelante, a sortear los obstáculos en la relación con sus nuevos entornos como una luz a través del túnel.

En cuanto a los aspectos que dificultan la adaptación, encontramos en la tabla los bajos niveles de autonomía, estados psicológicos depresivos, la no construcción de un sueño para el futuro y la no construcción de un proyecto adecuado a las condiciones del nuevo entorno y poca tolerancia al fracaso. Estos aspectos muestran en los niños dificultades para diseñar estrategias de resolución de problemas, para asumir responsabilidades consigo mismo y con los demás y para crear canales de comunicación efectiva en sus interacciones sociales. En el caso de los niños que presentan buenos niveles de autonomía y al mismo tiempo síntomas depresivos, se puede pensar que éstos sufrieron mucho con el desplazamiento y no han terminado de elaborar el duelo frente a las pérdidas que este hecho les significó, lo que puede considerarse normal.

A pesar de todas estas dificultades, podemos observar que todos los niños muestran un interés muy grande por organizar una relación afectiva con el nuevo ambiente porque presentan un sentimiento ético de cuidado hacia sí mismos, lo que implica cuidado del entorno, la prueba está en que todos valoran positivamente la escuela y en que asistieron por más de un año resolviendo diferentes dificultades, a los talleres 
que les planteamos, por ello podemos afirmar que estos aspectos dificultan la adaptación pero no la determinan.

Otro elemento que constituye la organización psicológica de los niños es su relación con el ambiente anterior al desplazamiento. En este caso, la tabla revela que la gran mayoría de los niños han construido vínculos muy estrechos con este ambiente, habían organizado su identidad personal con esos lugares, con las características de las redes sociales de los entornos en que intervenían, con los roles y las actividades que se desarrollaban allí frente al ambiente físico, rural y por tanto con la cultura campesina.

También se observa que la mayoría de niños, participaba de forma activa y gratificante en varios entornos al tiempo que desarrollaban actividades diversas en las que participaban diferentes personas en distintos contextos y, esta variedad, según BRONFENBRENNER (1987), favorece un desarrollo adecuado del sujeto e incide en la capacidad que tienen ahora lo niños para construir relaciones saludables con el nuevo ambiente.

Con relación al ambiente anterior, se observan dificultades (por ejemplo en el caso de $\mathrm{N}$ ) quien no encontró ninguna posibilidad de vida en este ambiente. Otros niños encontraron dificultades en sus entornos familiar, escolar y a nivel de su mesosistema lo que demuestra que todos los niños no perciben el ambiente anterior como un paraíso, como se suele creer; de hecho manifiestan conflictos en entornos vitales para su desarrollo, y varios de ellos no consideran que ese ambiente llenen sus expectativas de vida, percibiendo un futuro incierto en cuanto a permanecer en el mismo. Esta evaluación es un indicador adecuado de desarrollo y un factor positivo para la adaptación al nuevo entorno.

Con respecto al proceso adaptativo actual de los niños, encontramos que ellos aún no han elaborado una imagen integrada del ambiente actual porque apenas lo están conociendo y apenas están iniciando su proceso de aculturación. Podemos ver además que los niños están interesados en desarrollar este proceso y para ello han implementado varias estrategias como integrarse a las redes sociales presentes en los entornos en los que participan, conocer sus espacios físicos inmediatos y aprovechar sus propiedades para el juego o para cualquier otro tipo de diversión, poner en marcha sus planes para llevar a cabo sus proyectos de vida que en su mayoría se refieren al estudio, asumir sus responsabilidades laborales en forma positiva y transitoria como una forma de ayudar al bienestar propio y de la familia y como la posibilidad de conocer nuevas cosas, nueva gente, alternar sus actividades laborales con actividades placenteras, aprovechando su tiempo y los recursos que tienen a la mano como el televisor, el equipo de sonido y un libro agradable, el disfrute de las oportunidades que ofrece la cultura urbana con su tecnología y con sus espacios de juego y la facilidad para desarrollar las actividades laborales como la electricidad para cocinar, los medios de transporte que evitan las largas caminatas bajo el sol o la lluvia, propias del campo.

Otra estrategia muy importante implementada por los niños en su adaptación es el disfrute de las actividades que se hacen en familia que son muy importantes porque le permiten al niño adentrarse en la nueva cultura al mismo tiempo que fortalece su propia identidad. Todas estas estrategias le permiten apropiarse de los nuevos entornos y darle sentido a su vida formando parte de ellos. Así mismo, la tabla muestra aspectos que dificultan el proceso adaptativo. Entre estos, los más frecuentes son: la tendencia que tienen los niños a idealizar su relación con el ambiente anterior, pues muchos de ellos aún no han elaborado el duelo frente a diferentes aspectos de este ambiente, lo que es normal frente a lo reciente del desplazamiento. Sin embargo sería muy importante hacer un seguimiento a los niños para conocer qué tanto logran desvincularse de su ambiente anterior y hasta 
dónde este vínculo obstaculiza su proceso adaptativo.

Por otro lado, se puede observar que los niños poseen una limitada capacidad económica en los entornos para proveerlos a ellos y a sus familias de ayuda instrumental que garantice su supervivencia. En realidad las condiciones de vida en el ambiente receptor, son muy difíciles, por ello es muy importante el papel de la familia y de la red de apoyo de los niños por su capacidad de crear alternativas que le permitan sostenerlos material y emocionalmente.

Con respecto al ambiente, los niños construyen su adaptación especialmente con base en cuatro entornos: la familia, la escuela, los entornos recreativos y la iglesia. El papel de la familia en la adaptación de los niños es relativa en los casos en donde las relaciones intrafamiliares son armónicas, los niños perciben este entorno como plataforma y como un vehículo para su integración en los otros entornos; pero cuando se presentan conflictos intrafamiliares en los que los niños resultan maltratados física y psicológicamente, ese microsistema, pasa a ser el mayor obstáculo que afrontan los niños en su proceso de adaptación.

En cuanto a la escuela ha sido percibido por ellos como el entorno más importante para su adaptación puesto que en su interior es donde ellos pueden satisfacer todas sus necesidades de niño: jugar, aprender, conocer el mundo, participar en redes sociales con pares y con adultos que le proveen apoyo emocional, compañía y protección que a veces no puede brindarle la familia. Además el asistir a la escuela, le permite sentir a los niños que están haciendo algo para alcanzar sus sueños y para vivir mejor el futuro. Igualmente, por su carácter socializador, la escuela facilita el proceso de aculturación o de integración social.

En lo que tiene que ver con los entornos recreativos propiamente, constituidos en general por la cuadra, el parque, la cancha, etc, son considerados por los niños como vitales para su adaptación porque les permite participar en sistemas de pares que les brinda apoyo emocional, además de realizar diferentes actividades placenteras, exponer sus iniciativas y dar rienda suelta a su imaginación, comprendiendo poco a poco en la práctica, las nuevas normas sociales.

Se puede decir que la escuela, los entornos recreativos y en algunos casos la familia, son el dispensario en donde los niños se proveen de motivación, de fortaleza y de conocimientos para sortear las dificultades laborales, económicas y emocionales que se les presenta en el nuevo mesosistema.

Por último, la iglesia al ser de la misma doctrina de la que asistían antes de ser desplazados, constituye el punto de intersección entre ambos ambientes y por un lado, les permite a los niños y a sus familias conservar su identidad al mismo tiempo que les ayuda a integrarse al mismo orden social. Esto implica seguridad emocional; además la iglesia en sí misma es un vehículo integrador social alrededor de la fe, que evita el rechazo de y hacia las personas en situación de desplazamiento.

De igual manera, la iglesia impulsa, la organización de redes sociales de apoyo entre hermanos en la fe, muy potentes en su capacidad de proveer apoyo emocional e instrumental.

Se observa que el desplazamiento constituyó para los niños una transición ecológica por el cambio de entornos y de actividades específicas; también, que la mayoría de los niños asumen en el ambiente actual, prácticamente los mismos roles que asumían en el ambiente anterior, lo que indica por un lado, que la transición se dio a nivel del macrosistema el cual pasó de rural a urbano y por otro, que existen aspectos similares entre estas dos culturas al interior del país, esto implica un cambio de diseño del ambiente físico y por consiguiente de las actividades propias de cada microsistema las cuales varían en su contenido pero no en la estructuración de la vida cotidiana de los niños; 
es decir, en ambos ambientes los niños realizan actividades laborales, académicas, educativas y recreativas tanto en forma individual como grupal, sin embargo, es diferente el trabajo que realizaban en el campo cultivando la tierra o cuidando los animales, al que realizan en la ciudad como vendedores ambulantes, tenderos o amas de casa. Lo mismo sucede con las actividades académicas y recreativas.

Ésta similitud entre los patrones de actividades entre los mesosistemas anterior y posterior al desplazamiento, conllevan a una regularidad entre los roles que los participantes 'asumían en el campo y los que ocupan ahora. En ambos ambientes los niños asumen roles escolares, de compañeros de juego, de participantes activos en el grupo familiar y en la iglesia y de trabajadores, en este caso, el grado de responsabilidad es muy parecido al de los adultos.

Esta continuidad de los roles a través del desplazamiento, es un punto a favor para la adaptación porque le permite a los niños conservar el substrato psicológico de su vida cotidiana el cual puede ser llenado con su participación en las nuevas actividades, las cuales se constituyen en un canal de aculturación muy efectivo, al respecto, sería muy importante conocer qué tanto se mantienen estos roles cuando el desplazamiento indica un cambio radical entre culturas como es el caso de los grupos étnicos.

\section{CONCLUSIONES}

A partir de estos análisis pudimos llegar a las siguientes conclusiones: no es posible generalizar en cuanto a la forma en que los niños conciben su situación de desplazamiento y su proceso adaptativo, ya que pueden organizar tres tipos de percepción sobre dicho proceso: positiva, negativa y ambivalente. La positiva implica la valoración favorable del entorno actual y la detección de algunas falencias en su relación con el ambiente anterior por parte de los niños; la negativa se organiza cuando los niños no han logrado apropiarse de su ambiente actual, lo valoran negativamente y conservan una ilusión muy fuerte de regresar a su ambiente anterior; y, la percepción ambivalente, se refiere a una valoración del ambiente actual y del ambiente anterior que presenta aspectos muy positivos y muy negativos que incluso los mismos niños no saben cual de los ambientes prefieren.

Por otro lado, los aspectos considerados por los niños como favorables para su proceso de adaptación son: las redes sociales de apoyo (la familia, la escuela y los sistemas de pares), los recursos propios de la ciudad (la electricidad, los carros, los parques de diversiones y el poco esfuerzo físico que requieren los oficios de la ciudad comparados con los del campo) y la posibilidad que les ofrece el nuevo entorno para realizar sus proyectos de vida. Así mismo, los aspectos desfavorables más mencionados por los niños son: pérdida de los objetos de apego a raíz del desplazamiento, el exosistema actual y las dificultades económicas que encuentran en el entorno donde habitan ahora.

También es claro que la familia no siempre es un factor positivo para la relación entre el niño y su ambiente actual y que la escuela se convierte en un contexto primario para el proceso adaptativo de muchos niños.

Finalmente, también se pudo concluir que no todos los niños desplazados se convierten en víctimas irremediables de los traumas, de las pérdidas que tal evento les ha significado. Por el contrario, encontramos que la gran mayoría de los niños que trabajaron con nosotros crean diversas estrategias, unas más eficaces que otras para darle sentido a sus vidas en los nuevos entornos.

\section{RECOMENDACIONES}

Sería muy importante abordar a fondo en un estudio similar, (además de la escuela y la familia) los microsistemas recreativos, religiosos y laborales puesto que permiten un co- 
nocimiento más concreto de la relación entre el niño y su nuevo ambiente al igual que es necesario la elaboración de un diseño longitudinal que permita hacer un seguimiento al proceso adaptativo de estos niños, a través del tiempo. También es muy útil, siguiendo las recomendaciones de MARTÍNEZ, MAYA y GARCÍA (2000), realizar un estudio riguroso sobre el papel que cumplen las redes primarias de apoyo, ya sean la familia, la escuela, el sistema de pares u otros, en el proceso adaptativo del niño

Por último, consideramos que esta capacidad e interés de los niños para salir adelante a pesar de todas las pérdidas que han sufrido y de las circunstancias de vida tan difíciles que deben afrontar ahora, son los mayores recursos que deben tenerse en cuenta en el diseño de programas de intervención en salud mental, orientados a mejorar la calidad de vida, tanto en los niños en situación de desplazamiento como en sus familias y en definitiva con cualquier comunidad que conviva con situaciones adversas; construir estrategias de intervención que se basen en una concepción de los niños como sujetos capaces, competentes, agentes de su propia adaptación y no como víctimas u objetos de su trágico destino.

\begin{abstract}
This article describes the perception of a group of displaced children concerning their relationship to their current environment and the factors that favor or disfavor it. This is an exploratory study that analyzes the participating children's self-perception, their relationship to their previous and current environments and the roles that family and school play in this relationship, taking into account our observations, cognitive maps, life stories and interviews with their parents and teachers. Not all children consider homelessness and the adaptation process as negative factors. Finally, we conclude that it is not possible to set a fixed attitude for children with respect to their homelessness and their new social context.
\end{abstract}

Key-words: ecological transition; ecology and development; displaced children; adaptation.

\section{REFERENCIAS BIBLIOGRÁFICAS}

AMAR, A.J. Pobreza, resiliencia y aprendizaje infantil. Universidad del Norte. Fundación Bernard Van Leer, 1999. Disponible en Internet: http://www.worldbank.org/ children/nino/basico/ amar.htm

BRONFENBRENNER, U. Ecología del desarrollo humano. Buenos Aires: Paidós, 1987.

CODHES. Informe sobre Desplazados en Colombia y el impacto de la Ayuda Estadounidense. En: Desplazados, rostros anónimos de la guerra, 2001. Disponible en Internet: http://www.colombiapolicy.org/ codhes.htm
GONZALEZ, B.M. El desplazamiento forzado en Colombia. Médicos sin Fronteras España. Santafé de Bogotá, Colombia, 2001. Disponible en Internet: http:/ www.msf.es/paises/MSF44.pdf

GUTIERREZ, D. Acerca de la repercusión de la violencia política en los niños (escolares primarios) desplazados. En: ROJAS, J.E. (Org.). Desplazamiento, derechos humanos y conflicto armado. Colombia: CODHES, 2000.

HOLAHAN, Ch. Psicología ambiental (Enfoque General). México: Limusa, 2001.

KAMII, C. La teoría de Piaget y la educación. En: ÁlVAREZ, A. (Org.). Psicología y educación. Realizaciones y tendencias 
actuales en la investigación y en la práctica. Madrid: Visor Distribuciones, 1987.

MARTÍNEZ, M.F.; MAYA, M.; GARCIA, C. La integración social de los inmigrantes desde los recursos sociales naturales. Facultad de Psicología. Universidad de Sevilla, España, 2000. Disponible en Internet: http://www.opi.upco.es/otros\%20 documentos/congresso/datos/CDRom/ Integración/Ponencia/Manuel Fco Mtnez Maya Jariego.pdf

PALACIO, J. Estrés post-traumático y resistencia psicológica en jóvenes desplazados. Centro deInvestigación en Desarrollo Humano y el laboratorio de Psicología del Desarrollo de la niversidad de París X -Nanterre, 1999. Disponible en Internet: www.uninorte.edu.co/divisiones/ humanidades/opsiun/articulo3.htm

ROJAS, J.; ROMERO, M. Conflicto armado y desplazamiento interno en Colombia. En: Esta guerra no es nuestra. Niños y desplazamiento forzado em Colonbia. Bogotá: CODES-UNICEF, 2000.

UNICEF, Colombia. Niñez y conflicto armado, 2001. Disponible en Internet: http:// www.unicef.org.co/ 03c_f.htm

Recebido em 30/03/2003 Modificado em 01/05/2003 Aprovado em 10/05/2003 